\title{
ULTRASOUND AND CT REGISTRATION QUALITY: ELASTOGRAPHY VS. CLASSICAL B-MODE
}

\author{
Matthias Keil, Member, IEEE ${ }^{1}$, Philipp J. Stolka ${ }^{2}$, Marion Wiebel ${ }^{3}$, \\ Georgios Sakas ${ }^{1}$, Elliot R. McVeigh ${ }^{4}$, Russell H. Taylor ${ }^{2}$, Emad Boctor ${ }^{5}$
}

\begin{abstract}
Ultrasound and computed tomography registration is a valuable tool for interventional imaging and navigation, but current methods are limited in reliability or usefulness, requiring large fields of view, large ultrasound penetration depth, distinct image features, or contours of the investigated organs. Computationally cheap, smallfield-of-view registration proved elusive so far. We propose the use of interventional ultrasound elastography imaging to perform limited-field-of-view, limited-features registration reliably, examining the image acquisition and registration in the example application of partial nephrectomies. Furthermore, the benefits of elastography vs. conventional B-Mode ultrasound imaging are investigated.
\end{abstract}

Index Terms - computed tomography, elastography, B-Mode ultrasound, kidney, rigid registration

\section{INTRODUCTION}

Multi-modality ultrasound (US) and computed tomography $(C T)$ registration is a valuable tool for interventional imaging and navigation. However, current methods suffer from a number of limitations reducing the registration's reliability or usefulness. To yield reliable results, requirements include a large field of view (FOV), a large ultrasound penetration depth (around $10 \mathrm{~cm}$ ), many distinct image features, and possibly outer contours of the organs to be imaged. Furthermore, US and CT "don't speak the same language", i.e. while one shows acoustic impedance gradients, the other yields radiation opacities. One approach to overcome this second problem is to adapt one modality to the other, e.g. by computing pseudo-US from CT data to register it to the original US [1], [2]. However, small-FOV registration proved to be a challenging and exigent task so far.

In the present work, we propose to use elastography imaging $(E I)$ as a tool to enable limited-FOV, limited-features US-to-CT registration without expensive computations. The application under investigation is partial nephrectomy, as this intervention does not allow the acquisition of a large FOV, surrounding features are unstable due to kidney motion during the operation, and thus one has to rely on internal structures and use EI to acquire these. Here, we aim to show that, compared with B-Mode US, EI is a promising approach for solid organ registration with CT.

\footnotetext{
${ }^{1}$ Fraunhofer IGD, Department A7, Cognitive Computing \& Medical Imaging, Fraunhoferstrasse 5, D-64283 Darmstadt, Germany.

${ }^{2}$ Johns Hopkins University, ERC-CISST/Center of Computer-Integrated Surgical Systems and Technologies, 3400 N. Charles Street 113 CSEB, Baltimore, MD 21218, USA.

${ }^{3}$ TU Darmstadt, Graphisch-Interaktive Systeme, Rundeturmstrasse 10, D64283 Darmstadt, Germany.

${ }^{4}$ Johns Hopkins University, Department of Biomedical Engineering, Baltimore, MD 21218, USA.

${ }^{5}$ Johns Hopkins Medical Institutions, DMIP/Division of Medical Imaging Physics, 601 North Caroline Street JHOC, Baltimore, MD 21287, USA.
}

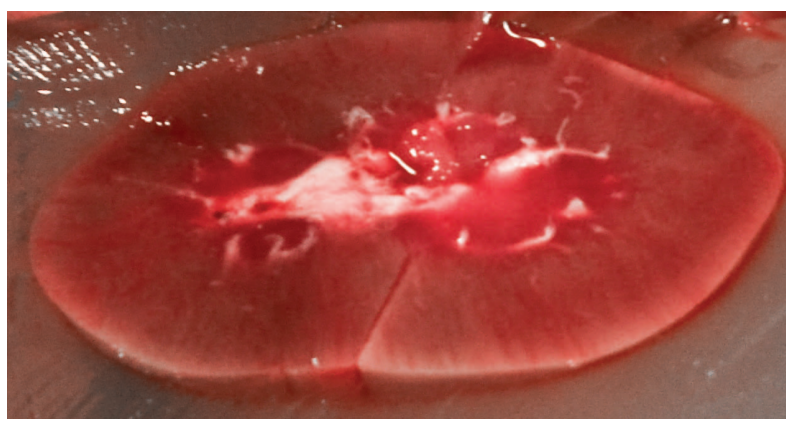

Fig. 1: Cross section of a porcine kidney embedded in gelatin, exhibiting the relevant internal structures (renal pelvis, calyces, surrounding tissue)

After describing the phantoms and data acquisition procedures (sec. 2), the registration approach is broken down into its algorithmic components (sec. 3). A series of registration experiments (sec. 4) together with its results (sec. 5) is concluded with a discussion of their relevancy and future work (sec. 6).

\section{DATA ACQUISITION}

For the registration experiments, three distinct datasets based on kidney phantoms had to be acquired. These included CT (contrastenhanced), ultrasound B-Mode, and ultrasound elastography (EI).

\subsection{Phantoms}

For these preliminary investigations, ex-vivo phantoms (animal tissue preparations exhibiting structures resembling actual in-vivo conditions) were chosen as experiment materials. These phantoms were based on porcine kidneys (extracted shortly after exitus) due to their similarity to the human organs. The kidneys were partially treated with arterially and per urethra applied CT contrast agent (5\% suspension of $\mathrm{TiO}$ powder $(5 \mu \mathrm{m})$ in agar solution). Embedded in a stabilizing porcine gelatin mold, the kidneys could then be subjected to the CT and US scanning protocols.

Additional fiducials - from synthetic alginate injections (Kromopan 100) - were inserted into the phantoms to facilitate later registration. However, they were only used to visually validate the registration correctness in the presented experiments.

\subsection{Ultrasound Elastography}

Ultrasound elastography is a non-invasive imaging method in which the elastic properties of tissues can be used to detect or classify lesions [3]. Since cancerous lesions are up to 30 times stiffer than 


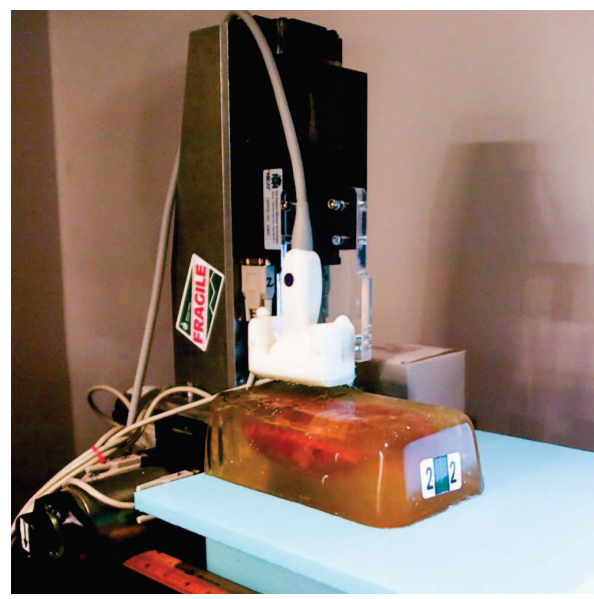

Fig. 2: US scan setup, with probe (white) held by the robot

normal soft tissues, this elastic property can be used in EI to differentiate tumors from surrounding normal tissues. By comparing the US radio-frequency $(R F)$ data of kidney phantoms before and after compression, strain images can be generated using cross-correlation algorithms. Stiffer lesions tend to generate lower strain values when compared to the softer surrounding tissues. Thus, this mode of imaging provides a novel view of the tissue that is otherwise not available using the traditional B-Mode ultrasound.

To collect ultrasound data, we used an Antares ultrasound system (Siemens Medical Solutions, USA) with a high frequency ultrasound transducer (VF13-5). The ultrasound images were obtained with depth of $6 \mathrm{~cm}$ and pixel size of $0.1132 \mathrm{~mm}$. Access to RF data was obtained through a User Research Interface provided by Siemens. Also, access to Siemens real-time elasticity imaging (eSie Touch) was granted.

In order to provide reliable and reproducible volume information, the ultrasound data was sampled with a robot-based system, yielding a series of planar-parallel B-Mode and strain slices through the respective phantom (fig. 3). This setup should be similar to $3 \mathrm{D}$ probes. After manual selection of the scanning region (to contain sufficiently descriptive features), the phantoms were positioned within the workspace of a high-precision three degrees-of-freedom $(D O F)$ cartesian robot (DMC-21x3 with three servo motor stages, by Galil Motion Control; repeatability better $0.01 \mathrm{~mm}$ ). Balancing dense data requirements and low elevational resolution of the ultrasound probe, the stage translated the probe to new positions every $\Delta_{Y}=2 \mathrm{~mm}$ apart, then performed five repeated compressiondecompression cycles (amplitude $d_{Z}=3 \mathrm{~mm}$ (approx. 5\% strain), period $T=2 s$ ), during which ultrasound data was sampled (at $16 \mathrm{~Hz}$ ). From these, one representative maximum decompression pair was chosen manually to obtain slice data.

\subsection{Computer Tomography}

In actual image-based renal interventions, contrast-enhanced CT can be performed at different times after contrast agent injection, yielding different information about the region of interest: within a few minutes after arterial injection ("early phase" with highlighting of the renal cortex), after a few minutes more ("delayed phase" after the contrast agent has been diffused into the collective system, highlighting the calyces), or in-between (showing the venous system).
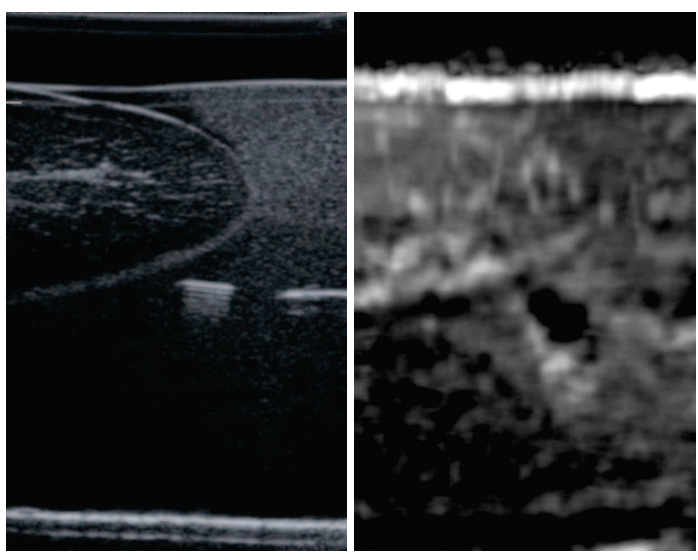

Fig. 3: B-Mode ultrasound (left) and elastography (right) slice of kidney phantom. Note the different definition of markers and renal structures.

For these experiments, contrast agent was injected so it filled both the arterial and the collective vasculature.

We have access to a Toshiba CT scanner (Aquilion 320) that can provide a full scan with $0.5 \mathrm{~mm}$ resolution in less than a second.

\section{REGISTRATION APPROACH}

The goal of our work is to register clinical ultrasound and CT datasets of kidneys. To test the quality of our registration approach and to enable us in comparing the properties of USEI and B-Mode ultrasound volumes in a more standardized way, we are using the phantoms mentioned above for registration.

Our registration application is based on the open source development toolkits MITK from the German Cancer Research Center (DKFZ), ITK and VTK from Kitware Inc., as well as Qt from Qt Software. Using these toolkits enables working with standardized, well established algorithms.

The registration approach can by classified according to the metric, the optimizer and the transformations being used for registration of the datasets [4]. Based on this information we describe the experiments that were performed.

\subsection{Metric}

One of the most important characteristics of a registration approach is the metric or cost function that is being used to compute the similarity of overlapping regions in the two volumes and therefore the quality of registration.

We chose Mattes mutual information [5] as our metric to overcome the problems that might arise when registering different modalities. This was necessary as there are big differences in tissue representation between B-Mode/USEI and CT image data. This characteristic prevents simpler metrics, which work on the image intensity values directly (e.g. mean squares computation), in giving accurate and representative estimates for the possible tissue combinations.

The similarity measure $S$ computation with Mattes mutual information is a function of the transformation parameters $\mu$. 


$$
S(\mu)=-\sum_{\iota \in L_{T}} \sum_{\kappa \in L_{R}} p(\iota, \kappa ; \mu) \log \frac{p(\iota, \kappa ; \mu)}{p_{T}(\iota ; \mu) p_{R}(\kappa)}
$$

It is based on histogram generation for both moving and fixed image. These histograms are built based on sampled voxel values and approximate the joint and marginal discrete probability distributions $p, p_{T}$ and $p_{R}$. We initialized the metric computation to choose 10,000 samples that were sorted into 24 bins. As the moving image is transformed during the registration process, linear interpolation was used to compute non-grid sample values.

Two representative metric plots can be seen in figs. 6 and 5 .

\subsection{Optimizer}

The optimizer evaluates the similarity metric while searching the parameter space of the registration. Given the rough cost hypersurface formed by the metric (cf. figs. 6 and 5), no direct gradient descent procedure could be guaranteed to find the global optimum. Furthermore the global optimum is entrenched in a steep valley, so would necessitate quickly changing the stepsize. Thus, methods like stochastic optimization (e.g. simulated annealing), genetic algorithms, or exhaustive search should be used to search the parameter space.

In the first instance we chose a Nelder-Meade downhill simplex optimization algorithm ("Amoeba" in ITK). Like other global optimization algorithms, this optimizer can be trapped in local optima or fail due to early convergence at one of the transformation parameters. Therefore it is usually necessary to adapt the parametrization of the optimizer itself to the given registration problem and the strategy that is implemented.

The Amoeba parameters that can be modified are the initial size of the simplex around the origin (simplex delta) and the convergence tolerances for the parameters under investigation as well as for the metric values being evaluated. The optimizer is then run for a predefined number of iterations or until convergence, whichever comes first.

The simplex size was initialized to values between 2 and 6 (cf. section 4), the parameter convergence tolerance set to 0.01 , function convergence tolerance to 0.001 , and maximum iterations to 200 .

\subsection{Transformation}

The parameter or search space of the optimizer for a given registration problem is defined by the admissible transformation type $M$ that has been chosen. The image data acquisition described in sections 2.2 and 2.3 constrained the orientation of the phantoms, so that only translational differences occur in the datasets. This defines a search space $M \subset \mathbf{R}^{3}$.

The main advantage of translational registration is that it is easy to keep track of the steps being taken by the optimizer to explore the search space. This makes a comparison between USEI and B-Mode registration possible.

Nevertheless, we have to admit and keep in mind that possible deformations due to strain application for USEI computation cannot be compensated just by rigid translation transformations.

\section{EXPERIMENTS}

The experiments described in this section were performed using subvolumes cropped from the EI, B-Mode, and CT volumes. The

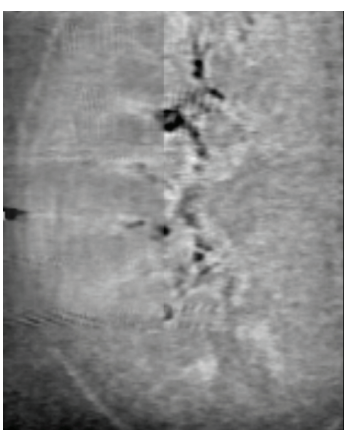

(a)

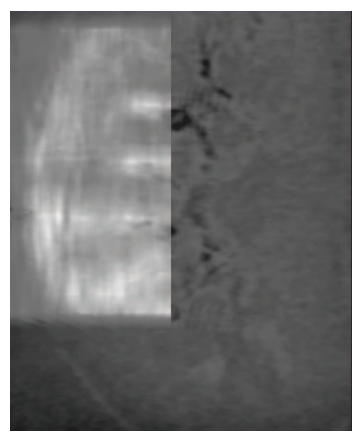

(b)
Fig. 4: Cross section showing the initial alignment of the EI (left) and CT volume (right). Note that the medulla as an internal structure of the kidney can be clearly seen in both modalities. To emphasize the structures in the respective modality, in each case the opacity of one of the datasets was reduced.

\begin{tabular}{cccc} 
& Global Optimum & Mean & Variance \\
\hline EI & -0.0128 & -0.0027 & $1.5487 e^{-6}$ \\
B-Mode & -0.0064 & -0.0016 & $4.6642 e^{-7}$
\end{tabular}

Table 1: Comparison of the Mattes mutual information metric for registration of CT and EI vs. B-Mode. The mean and variance measures were computed on the cost function (fig. 5 and 6)

subvolumes had a size of $34 x 29 x 64 \mathrm{~mm}^{3}$ for ultrasound and $72 \times 29 \times 90 \mathrm{~mm}^{3}$ for CT, with the US subvolumes contained in the CT subvolume. We chose the region of interest based on the expected amount of data for our clinical application. As only a small portion of the kidney is scanned during the operation, the volume is comprised of almost only kidney tissue. A cross section through the overlapping strain and CT volumes can be seen in fig. 4 .

In order to facilitate a comparison between the registration properties of strain images and B-Mode images, we chose to register both datasets with our CT volume using different initial offsets from a manually defined gold standard based on the fiducials and kidney boundary. We tested single displacements along the $x$ and $y$ axes as well as all pertubations of translation by $2 \mathrm{~mm}$ and $4 \mathrm{~mm}$ in positive and negative directions in all three dimensions. The last test was pertubing by a positive and negative displacement of $2 \mathrm{~mm}$ along the $x$ and $y$ axes and a $4 \mathrm{~mm}$ displacement in the $z$ direction, respectively.

With the general restrictions for the use of ex-vivo tissue for elasticity imaging, the porcine kidney phantoms exhibited both very imaging-friendly properties (clearly distinct surrounding material, stable geometry) and non-natural behavior (little to no temporal or repetition elasticity phenomena due to missing tissue perfusion and turgor) at the same time. Still, the geometry and availability of features in these physiological phantoms approximated the in-vivo case.

\section{RESULTS}

Registration success was measured by visual examination using the fiducials that were added to the phantom. These were clearly visi- 


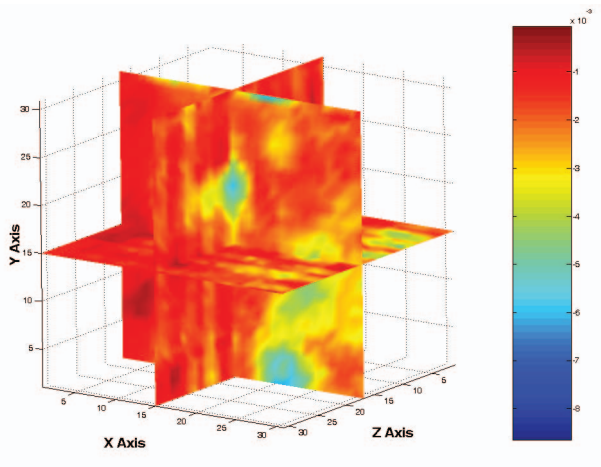

Fig. 5: Cost function surfaces for B-Mode/CT registration. Note the multitude of local minima.

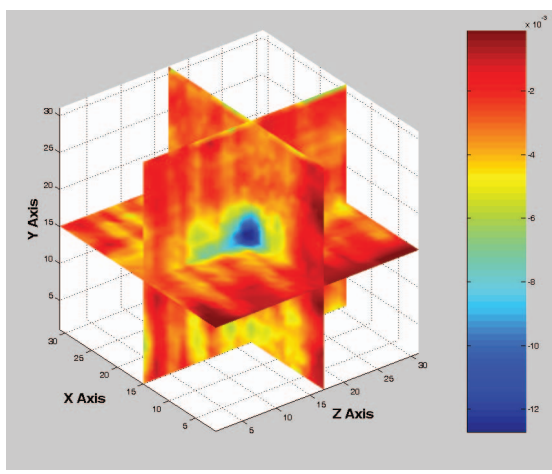

Fig. 6: Cost function surfaces for strain/CT registration intersecting at the global optimum $(15,15,16)$

ble in strain, CT and B-Mode images. Besides the fiducials a comparison with the information about the similarity measure and the transformation output of the optimization algorithm were used for monitoring.

For getting correct registration results for the B-Mode case, the registration parameters often had to be optimized very specifically to the given problem. For example, in testing the possible translations in $y$ direction, the Amoeba simplex had to be initialized with delta values (sizes) of 0 except for the $y$ direction, otherwise the optimizer failed and got stuck in local optima. Limiting the delta of the optimizer so radically narrows down the search space to one dimension and is very unrealistic. This behavior can be explained by having a look at the cost function plot of the B-Mode data (cf. fig. 5) and table 1. As there are several local minima having equal of even smaller (i.e., better) values for the metric, it is hard for the Amoeba optimizer to find the minimum of interest.

The situation is much different for the strain to CT image registration. As can be seen in fig. 6, the cost function has only one very pronounced global optimum. It is therefore much easier for the optimizer to find this optimum. However, we have to admit that this global optimum can also be missed by the Amoeba optimizer under certain conditions (e.g. intialization of the simplex fully on the plateau).

In order to account for the different imaging modalities, preliminary experiments with other metrics were performed as well.
In particular, noting that B-Mode US shows gradients of acoustic impedance, the gradient of the CT data was computed to match this property. Then, both images were registered using a mean-squares error metric. However, this approach proved less effective and produced even larger registration errors than the MI-based approach discussed in the present paper, in spite of the tissue representation being more similar in the two modalities then.

These results show that the metric used for the chosen registration algorithm is very well-suited for estimating the similarity between CT and EI images, even for the small field of view given in our test data.

\section{DISCUSSION}

We identified a metric that successfully measures the similarity between EI and CT images. It can be used for further investigations of the registration properties of EI and CT. We showed that the tissue representation between EI and CT is more similar and therefore EI registration outperforms B-Mode. For future work we are planning to evaluate more similarity measures using our protocols and to use an optimizer that has the ability to find the global optimum with a higher probability. Using this new optimizer we will approach nonrigid registration, to overcome the resulting registration errors that are due to possible tissue deformations from strain application. The goal of this paper was to show that it is possible to register EI and CT image data, which we did by comparing the registration results using well accepted registration techniques.

\section{ACKNOWLEDGMENTS}

This work was partially funded by the National Science Foundation under cooperative agreement NSF ERC-CISST EEC 9731748. Further support was provided by internal funds of the Johns Hopkins University and by the Fraunhofer Gesellschaft within the PROFIL program. Equipment support was generously provided by Siemens Medical Systems (Antares ultrasound) and the ERC-CISST (robotic stage).

\section{REFERENCES}

[1] Ramtin Shams, Richard Hartley, and Nassir Navab, "Real-time simulation of medical ultrasound from ct images," in International Conference on Medical Image Computing and ComputerAssisted Intervention (MICCAI), New York, USA, Sept. 2008.

[2] W. Wein, A. Khamene, D. Clevert, O. Kutter, and N. Navab, "Simulation and fully automatic multimodal registration of medical ultrasound," in MICCAI 2007 Proceedings. Oct. 2007, Lecture Notes in Computer Science, Springer.

[3] J. Ophir, I. Cspedes, H. Ponnekanti, Y. Yazdi, and X. Li, "Elastography: A quantitative method for imaging the elasticity of biological tissues," Ultrasonic Imaging, vol. 13, pp. 111-134, 1991.

[4] J. B. Antoine Maintz and Max A. Viergever, "An overview of medical image registration methods," Tech. Rep., In Symposium of the Belgian hospital physicists association ( $\mathrm{SBPH}-$ BVZF, 1996.

[5] David Mattes, David R. Haynor, Hubert Vesselle, Thomas K. Lewellyn, and William Eubank, "Nonrigid multimodality image registration," Medical Imaging 2001: Image Processing, vol. 4322, no. 1, pp. 1609-1620, 2001. 